\title{
Supplemental Information - Strained polymer thermal conductivity enhancement counteracted by additional off-axis strain
}

Brian F. Donovan, ${ }^{*}$ Ronald J. Warzoha, Tyler Cosby, Ashutosh Giri, Adam

A. Wilson, Andrew J. Borgdorff, Nicholas T. Vu, Eric A. Patterson, and

Edward P. Gorzkowski

E-mail: bdonovan@usna.edu 
A crucial aspect of this study is the actual measurement of the anistropic thermal conductivity of the polymers in question. As mentioned in the manuscript, this is a rather difficult measurement to make. In order to provide confidence in the use of Frequency Domain Thermoreflectance (FDTR) in making these measurements, we utilize well-known anistropic reference materials. The two reference materials are crystalline quartz and Highly Ordered Pyrolytic Graphite (HOPG), purchased from the MTI Corporation. Beyond confirmation of literature-based findings, $\frac{122}{2}$ we also include a comparison of these FDTR measurements to measurements taken with the somewhat more traditional Time Domain Thermoreflectance (TDTR). The use of both FDTR, which is critical to resolving our specific polymer samples as well as TDTR in resolving the anistropic thermal conductivity of our reference samples is intended to provide overwhelming confidence in the novel measurements presented in the manuscript. Additionally, we provide the MATLAB code used to analyze the data we have taken so that this process can be readily reproduced and expanded upon.

We examine the sensitivity of both techniques to both $\kappa_{r}$ and $k a p p a_{z}$. For these experiments, the pump and probe radii for TDTR are $\mathrm{w}_{0}=39.5 \mu \mathrm{m}$ and $9.1 \mu \mathrm{m}$, respectively. For these pump and probe diameters, we do not expect to be sensitive to thermal anisotropy via TDTR. Nevertheless, we supply sensitivity curves for each of our reference materials after measuring the thermal conductivity and fitting to the traditional analytical technique, where $\kappa=\sqrt{\kappa_{z} \cdot \kappa_{r}}$. TDTR results are supplied to confirm the effective results for FDTR. Literature values for effective thermal conductivity and anisotropic thermal conductivity are provided in Table S1 for each material below.

Table S1: Literature values for the thermal conductivity of the reference materials used to verify the efficacy of this technique. $\frac{112}{12}$

$\begin{array}{lccc}\text { Material } & \kappa_{\text {eff }}(\mathbf{W} / \mathbf{m} \cdot \mathbf{K}) & \kappa_{z}(\mathbf{W} / \mathbf{m} \cdot \mathbf{K}) & \kappa_{r}(\mathbf{W} / \mathbf{m} \cdot \mathbf{K}) \\ \text { X-cut Quartz (100) } & 8.6 & 6.2-6.9 & 10.4-11.2 \\ \text { HOPG } & 100-127 & 5-8 & 2,000\end{array}$


Fig. S1 shows the fit via TDTR for each of the corresponding materials listed above. The sensitivity to in-plane and cross-plane thermal conductivity is also plotted to the right of each fit to the normalized signal $(\Delta R / R)$ as a function of time delay.
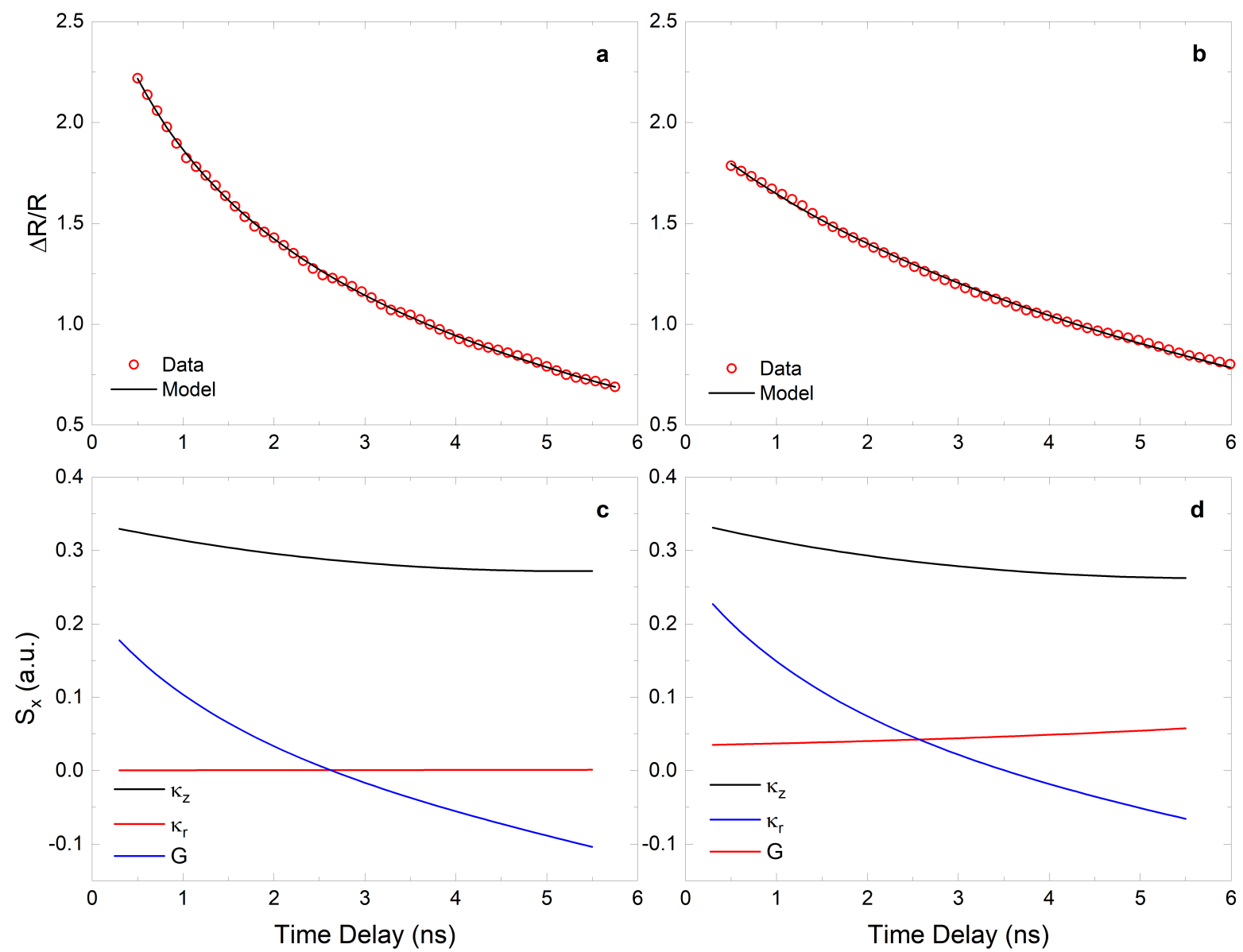

Figure S1: TDTR measurements of (a) X-cut Quartz (80 nm Al with $\mathrm{w}_{0}=65 \mu \mathrm{m}$ and $\mathrm{w}_{1}=9.9$ $\mu \mathrm{m}$ ) and (b) HOPG (80 nm Au with $\mathrm{w}_{0}=65 \mu \mathrm{m}$ and $\left.\mathrm{w}_{1}=9.9 \mu \mathrm{m}\right)$. Sensitivity to the throughplane $\left(\kappa_{z}\right)$ and in-plane $\left(\kappa_{r}\right)$ thermal conductivities, as well as the thermal boundary conductance between each metal transducer and respective substrate is provided in (c) and (d).

Our TDTR scans provide us with the through-plane thermal conductivity of each material; as shown, our scans are not sensitive to the in-plane thermal conductivity of each sample for a pump diameter of $\mathrm{w}_{0}=65 \mu \mathrm{m}$, a probe diameter of $\mathrm{w}_{1}=9.9 \mu \mathrm{m}$, and a modulation frequency of $f$ $=8 \mathrm{MHz}$. This is consistent with observations made by Schmidt et al. ${ }^{2}$ Values for through-plane thermal conductivity are $\kappa_{z}=6.22 \pm 1.3 \mathrm{~W} / \mathrm{m} \cdot \mathrm{K}$ for Quartz and $\kappa_{z}=6.88 \pm 1.1 \mathrm{~W} / \mathrm{m} \cdot \mathrm{K}$ for HOPG. These results are consistent with those found in Braun et al. ${ }^{1}$ and Schmidt et al. ${ }^{2}$ 
For our FDTR scans, we used two different pump sizes to verify our sensitivity to $\kappa_{r}$. Critically, we note that for some anisotropic materials we are sensitive to both $\kappa_{r}$ and $\kappa_{z}$ regardless of spot size, provided that we sweep through a sufficiently large range of frequencies. The reason we are insensitive to $\kappa_{r}$ for our polymer samples at large beam diameters is due to the fact that our film has a thickness on the order of the thermal penetration depth at lower frequencies; in this work, the polymers are $8 \mu \mathrm{m}$ thick. We refer the reader to our sensitivity curves for the polymer samples to gain insight into how sensitivity to $\kappa_{r}, \kappa_{z}$, and $\mathrm{G}$ changes as a function of the pump diameter.

We also note that obtaining in-plane thermal transport properties using a standard FDTR configuration is not a new practice. ${ }^{3-5}$ The radial thermal conductivity term is present in the heat diffusion equation, which is solved in two dimensions in the frequency domain via matrix inversion. 6

Our spot sizes were measured with a beam profiler (Thorlabs Model BP209-VIS) in knifeedge and scanning slit modes. The pump beam sizes used were $59 \mu \mathrm{m}$ and $16.8 \mu \mathrm{m}$, respectively, while the probe size remained fixed at $5.8 \mu \mathrm{m}$. For these measurements, we altered the pump size by placing different lenses ahead of the dichroic mirror used to steer the pump beam through our objective lens. Several tables summarizing our findings for $\kappa_{r}$ and $\kappa_{z}$ are provided below. Subsequent plots of frequency vs. phase and sensitivity for each measurement and sample are provided below the table. We note that the thermal conductivity of the $80 \mathrm{~nm} \mathrm{Au} / 5 \mathrm{~nm}$ Ti layer was determined using a four point-probe measurement in tandem with the Wiedemann-Franz law to be $\sim 160 \mathrm{~W} / \mathrm{m} \cdot \mathrm{K}$ at room temperature.

Table S2: Fit values for Quartz in different sensitivity regimes when $\mathrm{w}_{0}=16.8 \mu \mathrm{m}$.

$\begin{array}{lccc}\text { Fitting Condition } & \kappa_{e f f}(\mathbf{W} / \mathbf{m} \cdot \mathbf{K}) & \kappa_{z}(\mathbf{W} / \mathbf{m} \cdot \mathbf{K}) & \kappa_{r}(\mathbf{W} / \mathbf{m} \cdot \mathbf{K}) \\ 16.8 \mu \mathrm{m} \text { Pump, Full Fit } & 7.96 & 5.87 & 10.80 \\ 16.8 \mu \mathrm{m} \text { Pup, } f=1 \mathrm{kHz}-500 \mathrm{kHz} & 9.80 & 2.01 & 10.10 \\ 16.8 \mu \mathrm{m} \text { Pup, } f=500 \mathrm{kHz}-20 \mathrm{MHz} & 14.01 & 5.99 & 12.00\end{array}$

As shown in Table S3, we obtain the correct ratio for thermal anisotropy in quartz for our fitting routine over a full frequency range. To belabor the point, we also fit over two different frequency ranges where we expect to be sensitive to only $\kappa_{r}(f=1 \mathrm{kHz}-500 \mathrm{kHz})$ or $\kappa_{z}(f=500 \mathrm{kHz}-20$ 

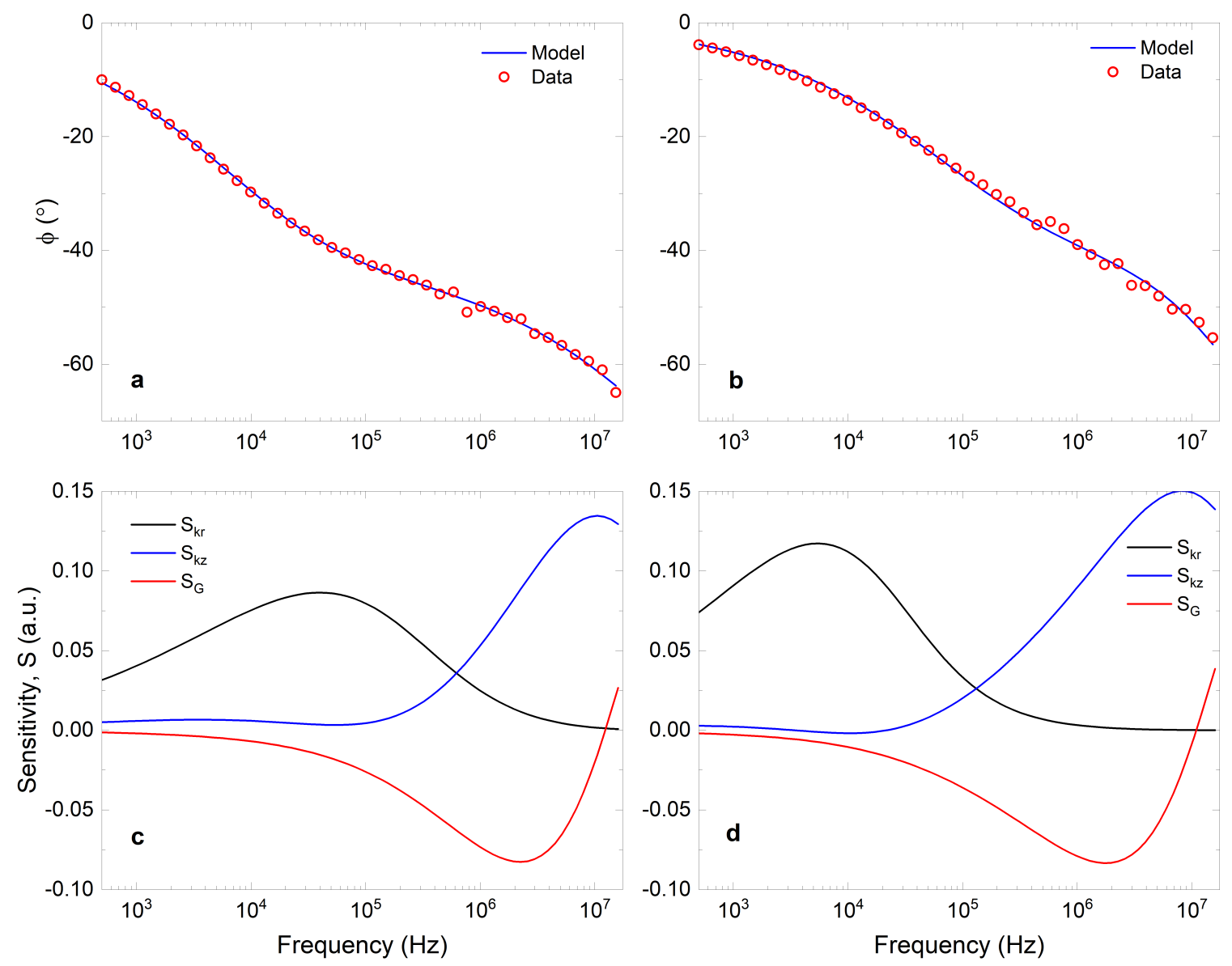

Figure S2: Fits and corresponding sensitivity for Quartz. (a,c) Pump $=59 \mu \mathrm{m},(\mathrm{b}, \mathrm{d})$ Pump $=16.8$ $\mu \mathrm{m}$

MHz) (see: Sensitivity plots in Fig. $S 3(\mathrm{~d})$ ). We note that for $\kappa_{e f f}$, we only fit to two parameters (i.e. $\kappa_{r}=\kappa_{z}$ ), while $\kappa_{z}$ and $\kappa_{r}$ are obtained via a 3 parameter fit. We see that when we fit over a range of $f=1 \mathrm{kHz}-500 \mathrm{kHz}$, we obtain a similar value of $\kappa_{r}$ when compared to the full fit, but we do not obtain a reasonable value for $\kappa_{z}$. Likewise, when we fit over our high frequency range $(f=500 \mathrm{kHz}-20 \mathrm{MHz})$, we have less sensitivity to $\kappa_{r}$, and the extracted value begins to diverge from its true value. However, the through-plane thermal conductivity is similar to what we obtained using the full fit. Thus, we confirm that our fit over the full frequency range provides us with sensitivty to both $\kappa_{r}$ and $\kappa_{z}$ according to Figs. S3 (c) and (d). We note that the values obtained for a $59 \mu \mathrm{m}$ pump were within $10 \%$ of all values listed in Table $\mathrm{S} 3$. 
To provide for additonal confidence and demonstrate the range of thermal anisotropy that can be resolved with FDTR, we also measure the in-plane and through-plane thermal conductivities of highly ordered pyrolytic graphite (HOPG). Our measured values of thermal conductivity are provided in the table below.

Table S3: Fit values for HOPG in different sensitivity regimes when $\mathrm{w}_{0}=16.8 \mu \mathrm{m}$ and $59 \mu \mathrm{m}$.

Fitting Condition

$16.8 \mu \mathrm{m}$ Pump, Full Fit

$16.8 \mu \mathrm{m}$ Pup, $f=1 \mathrm{kHz}-500 \mathrm{kHz}$

$16.8 \mu \mathrm{m}$ Pup, $f=500 \mathrm{kHz}-20 \mathrm{MHz}$

$59 \mu \mathrm{m}$ Pump, Full Fit

$59 \mu \mathrm{m}$ Pup, $f=1 \mathrm{kHz}-500 \mathrm{kHz}$

$59 \mu \mathrm{m}$ Pup, $f=500 \mathrm{kHz}-20 \mathrm{MHz}$ $\kappa_{e f f}(\mathbf{W} / \mathbf{m} \cdot \mathbf{K})$

175.64

204.79

173.86

139.55

102.73

119.68
$\kappa_{z}(\mathbf{W} / \mathbf{m} \cdot \mathbf{K})$

5.12

42.21

N/A

6.84

15.71

N/A
$\kappa_{r}(\mathbf{W} / \mathbf{m} \cdot \mathbf{K})$

2,240

1,833

N/A

1,930

575.80

N/A
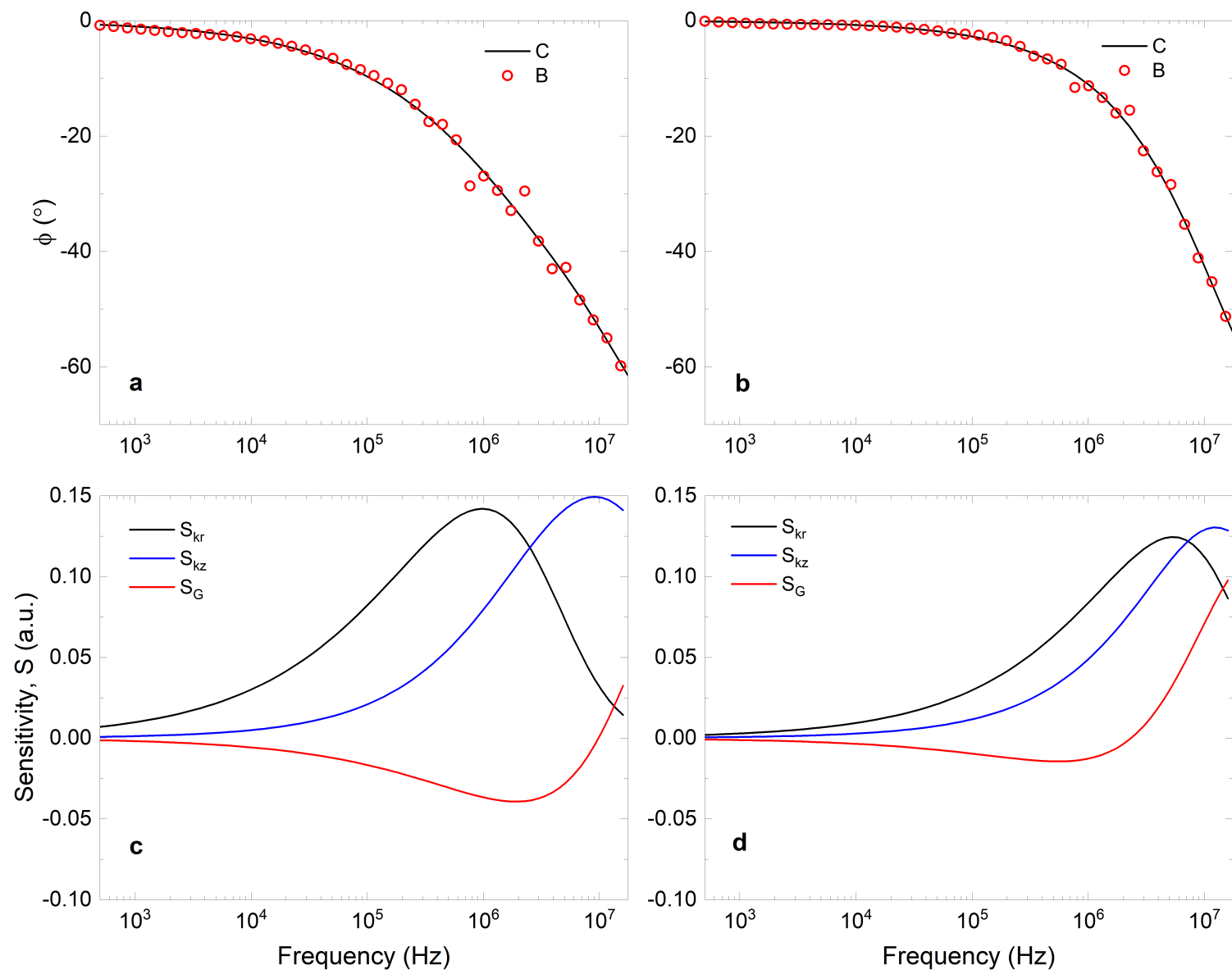

Figure S3: Fits and corresponding sensitivity for HOPG. (a,c) Pump $=59 \mu \mathrm{m},(\mathrm{b}, \mathrm{d})$ Pump $=16.8$ $\mu \mathrm{m}$ 
Here we are able to resolve both $\kappa_{z}$ and $\kappa_{r}$ when we fit over the entire range of frequencies at a pump diameters of both $\mathrm{w}_{0}=16.8 \mu \mathrm{m}$ and $59 \mu \mathrm{m}$. Without a fit over the full range of values, or apriori knowledge of the thermal boundary conductance, we can not independently resolve the in-plane and through-plane thermal conductivities simultaneously.

These results help to support our findings of thermal anisotropy in our polymer films by demonstrating that the technique is able to resolve a wide range of thermal anisotropy ratios, provided that a large enough frequency range is imposed on the pump beam for fitting to the phase lag at the sample surface. Because our films are suspended and range in thickness between 8 and $11 \mu \mathrm{m}$, we change the sensitivities to both in-plane and through-plane properties over different frequency regimes. As a result, we become more or less sensitive to in-plane thermal conductivity as increase or decrease the pump diameter. Critically, we are also able to resolve both $\kappa_{r}$ and $\kappa_{z}$ at our smaller pump size; our large diameter pump data is used for confirmation of $\kappa_{z}$ only. 
Below is the FDTR analysis code used in this work. Please note that this specific code was developed at the United States Naval Academy by US government employees and if used elsewhere should be accompanied with the appropriate attribution.

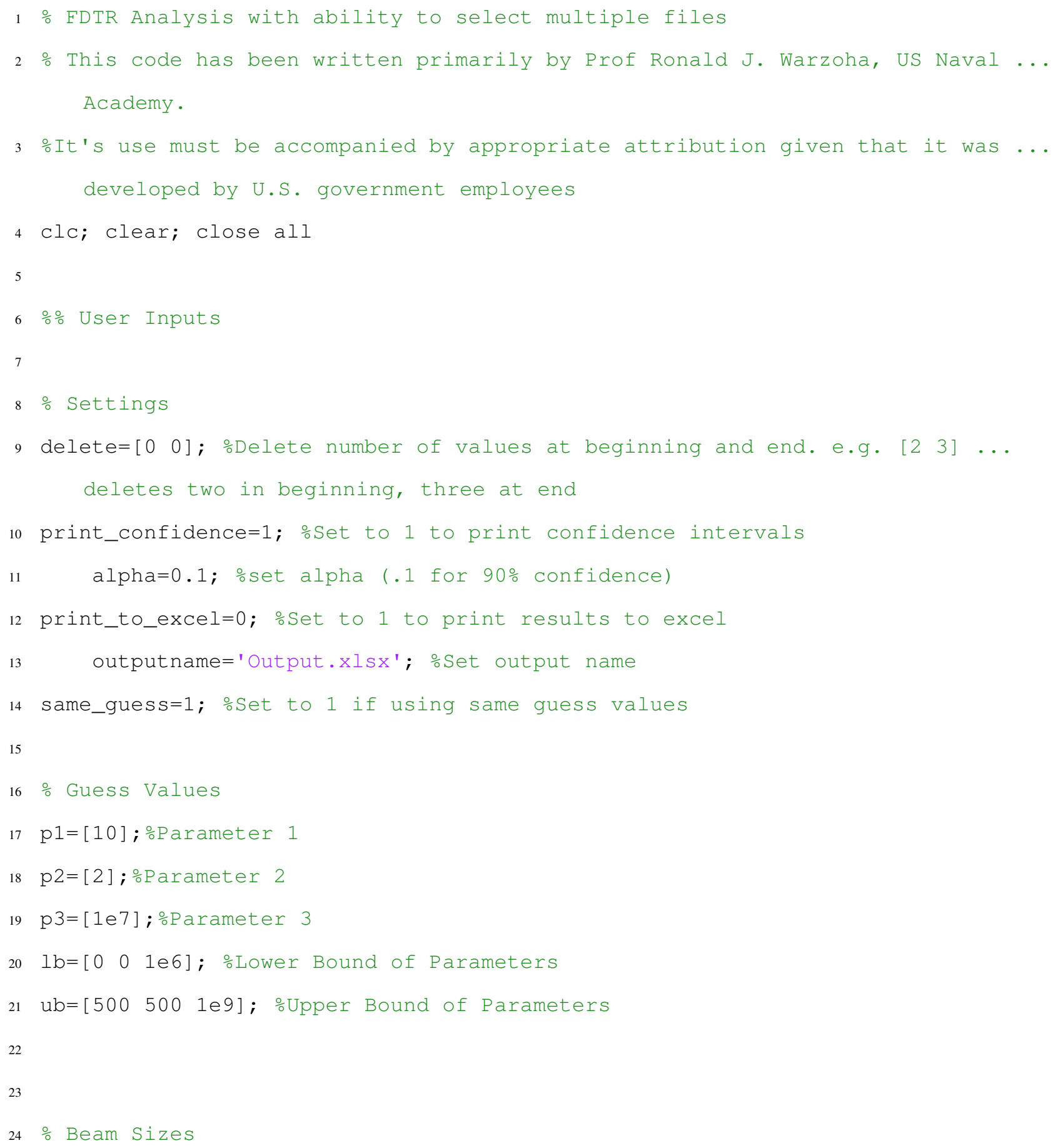




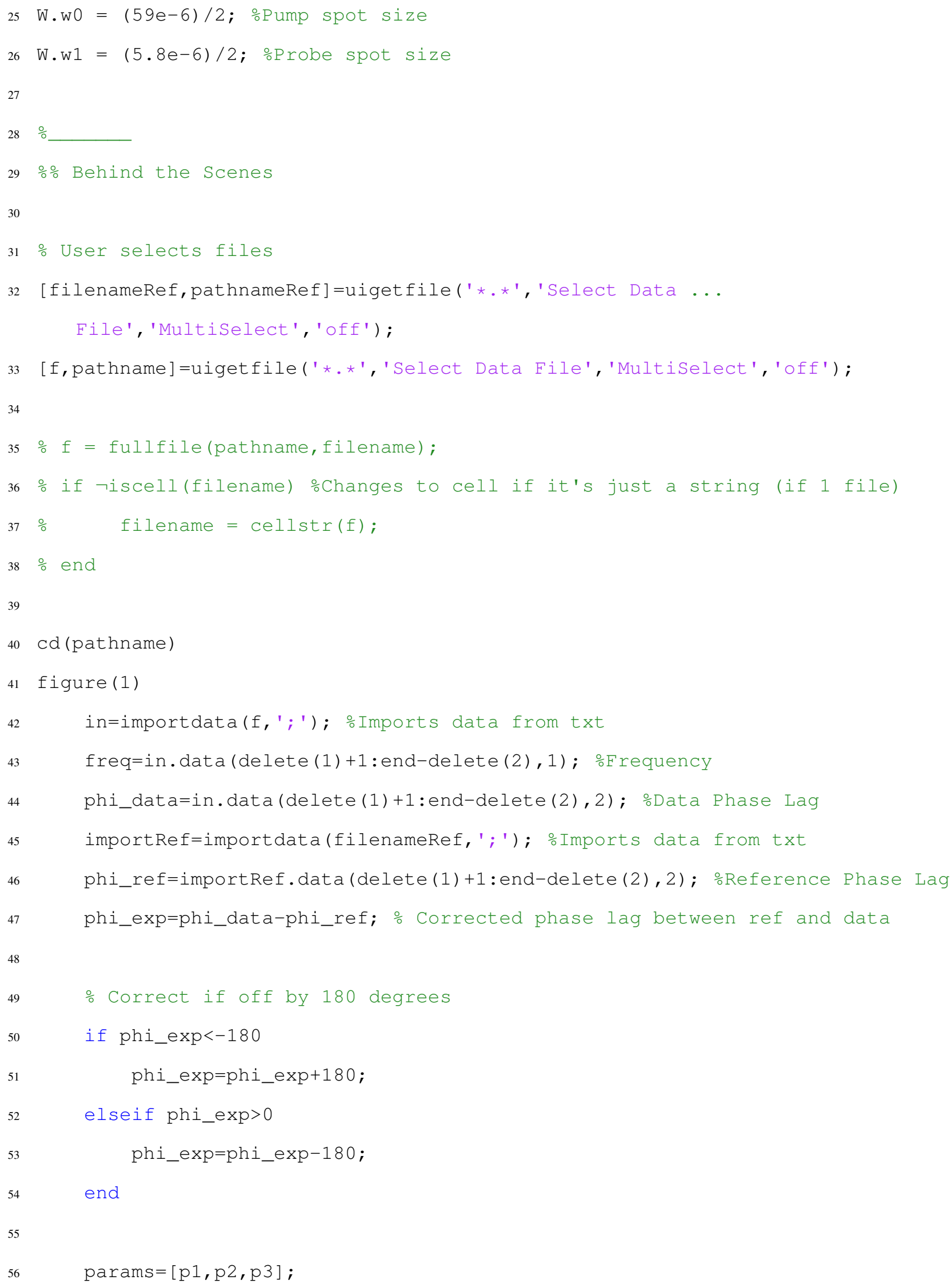

55 
\% Non Linear regression

[extparams, SSE] =fminsearch (SSEphifit, params, options);

fprintf ('Parameter $\backslash n k=\frac{\circ}{} \backslash n C=\frac{\circ}{\circ} \backslash n G=\circ e \backslash n$ ', extparams) ;

o Calculate function using the regressed parameters

[phi_model] =FDTR (extparams, freq, w) ;

\% Plot model

hold on

plot (freq, phi_model,'-r');

legend('Measured', 'Curve fit')

o Export formatted outputs to excel

if print_to_excel==1

output $1=[$ freq phi_exp phi_model];

header $=\{$ 'Freq' ' 'Exp', 'Model', ' ', 'Guess' $\}$;

xlswrite (outputname, filename, 'Al') ;

xlswrite (outputname, header, 'A2')

xlswrite (outputname, output1, 'A3')

xlswrite (outputname, params, 'E3') 


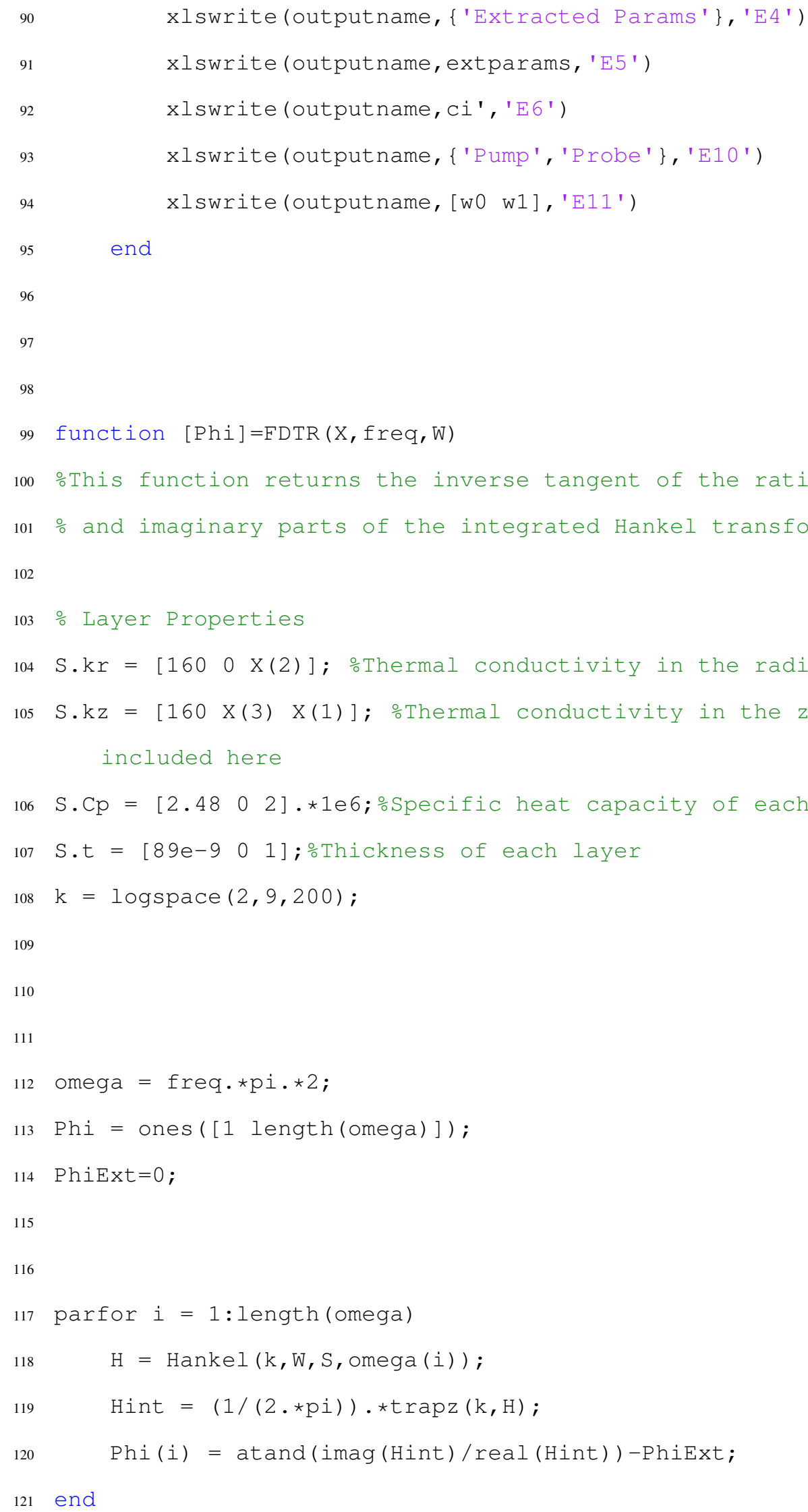

102

115

116 


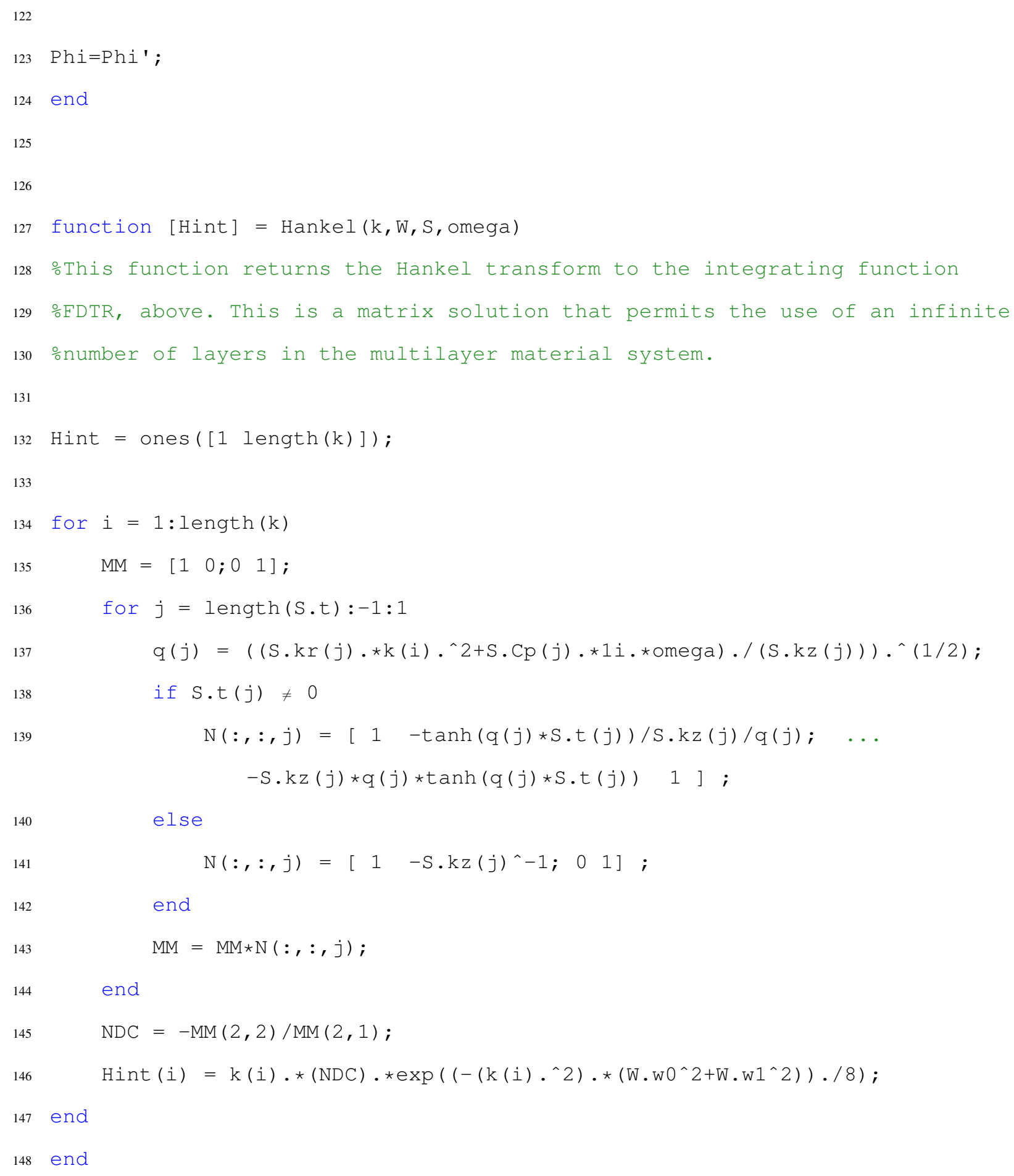




\section{References}

(1) Braun, J. L.; Olson, D. H.; Gaskins, J. T.; Hopkins, P. E. A steady-state thermoreflectance method to measure thermal conductivity. Review of Scientific Instruments 2019, 90, 024905.

(2) Schmidt, A. J.; Chen, X.; Chen, G. Pulse accumulation, radial heat conduction, and anisotropic thermal conductivity in pump-probe transient thermoreflectance. Review of Scientific Instruments 2008, 79, 114902.

(3) Medvedev, V.; Yang, J.; Schmidt, A.; Yakshin, A.; van de Kruijs, R. W. E.; Zoethout, E.; Bijkerk, F. Anisotropy of heat conduction in Mo/Si multilayers. Journal of applied physics 2015, $118,085101$.

(4) Yang, J.; Ziade, E.; Maragliano, C.; Crowder, R.; Wang, X.; Stefancich, M.; Chiesa, M.; Swan, A. K.; Schmidt, A. J. Thermal conductance imaging of graphene contacts. Journal of Applied Physics 2014, 116, 023515.

(5) Qian, X.; Ding, Z.; Shin, J.; Schmidt, A. J.; Chen, G. Accurate measurement of in-plane thermal conductivity of layered materials without metal film transducer using frequency domain thermoreflectance. Review of Scientific Instruments 2020, 91, 064903.

(6) Schmidt, A. J.; Cheaito, R.; Chiesa, M. A frequency-domain thermoreflectance method for the characterization of thermal properties. Review of scientific instruments 2009, 80, 094901. 